Alleinverdieners habe in der alten Gesetzesfassung implizit zugrunde gelegen. Die Erziehung von Kindern und die Pflege von Eltern oder Großeltern, so wurde unterstellt, seien Privatsache der Ärzte.

\section{Ärztinnen suchen verstärkt die Selbstständigkeit}

Mit den Anpassungen reagiert der Gesetzgeber auf eine veränderte gesellschaftliche Realität: Der steigende Anteil von Frauen unter den Ärzten macht sich zunehmend auch in den Praxen bemerkbar. Die Existenzgründeranalyse von apoBank und Zentralinstitut für die kassenärztliche Versorgung machte dies deutlich: $42 \%$ aller deutschen Ärzte, die die apoBank im letzten Untersuchungszeitraum 2008/2009 in die Selbstständigkeit begleitet hat, waren Frauen. 2000 waren es noch $37 \%$. Auffallend ist der Unterschied zwischen Ost und West. $40 \%$ der Existenzgründer waren im Westen weiblich, im Osten waren es sogar $58 \%$.

Die jetzt geschaffenen erweiterten Möglichkeiten sind ein Schritt hin zu einer größeren Flexibilität für Ärzte. „Auch Ärztinnen und Ärzte in eigener Praxis haben dadurch die Möglichkeit, sich eine Auszeit für die Familie zu nehmen“, urteilt Leutloff. „Die Freiberuflichkeit wird gestärkt.“

\section{Auch die finanzielle Seite gilt es zu bedenken}

Wer den Sprung in die Selbstständigkeit wagt, sollte bei der Familienplanung die finanzielle Seite im Blick behalten, rät Leutloff. Nach Angaben der apoBank finanzieren viele Existenzgründer ihre Praxen zum größten Teil über Kredite. Wer eine Auszeit für die Kinder nehmen will, sollte sich deshalb mit seinem Bankberater zusammensetzen und besprechen, wie sich die Auszeit auf die Finanzierung auswirkt und gemeinsam nach einer Lösung suchen. Außerdem: Wenn ein Vertretungsarzt tätig wird, läuft der Praxisbetrieb weiter, und die Honorare fließen. Wenn die Praxis gut organisiert ist, ist dies häufig eine lohnende Strategie. Und Elterngeld (maximal 1800 EUR pro Monat) gibt es auch für Freiberufler für einige Zeit zusätzlich vom Staat dazu.

Antonia von Alten, Neu-Isenburg

Medizinische Versorgungszentren

\title{
Die ärztliche Leitung wird in MVZ zur Pflicht
}

Mit dem GKV-Versorgungsstrukturgesetz plant die Regierungskoalition auch stärkere Restriktionen für Medizinische Versorgungszentren als bisher. Unter anderem soll die Rolle der Ärzte in den Zentren gestärkt werden. Manche der Neuerungen sind allerdings eher als kosmetische Maßnahmen zu werten.

$D^{\circ}$ er Anteil Medizinischer Versorgungszentren (MVZ) ist in den vergangenen Jahren stark gestiegen. Ende 2010 zählte die Kassenärztliche Bundesvereinigung mehr als $1.600 \mathrm{MVZ}$ in Deutschland, in denen mehr als 8.600 Ärzte überwiegend als Angestellte arbeiten. Binnen eines Jahres ist die Zahl der Ärzte in MVZ damit um mehr als 1.000 gestiegen.

Ein Ziel der Koalition ist es, in diesem stark wachsenden Sektor der ambulanten Versorgung den Einfluss der Ärzte zu stärken und so ein von ökonomischen Zwängen möglichst unabhängiges Arbeiten zu ermöglichen. Eine der Neuerungen, die der Entwurf des GKVVersorgungsstrukturgesetzes (GKVVStG) in der aktuellen Fassung vorsieht, ist daher, die ärztliche Leitung eines Medizinischen Versorgungszentrums (MVZ) rechtlich und faktisch auch in ärztlicher Hand zu legen. So heißt es im neu gefassten Paragrafen 95 des Fünften Sozialgesetzbuches (SGB V): „Der ärztliche Leiter muss in dem MVZ selbst als angestellter Arzt oder als Vertragsarzt tätig sein; er ist in medizinischen Fragen weisungsfrei“"

Gesundheitsökonom Jens Leutloff von der Deutschen Apotheker- und Ärztebank (apoBank) begrüßt diese neue Regelung: „Ärzte und nicht Kapitalinteressen haben das Sagen in der Versorgung." Diese Tendenz des Gesetzgebers zeigt sich auch darin, dass eingeschränkt werden soll, wer ein MVZ gründen darf. Aktiengesellschaften dürfen danach künftig keine MVZ mehr errichten. Sie können nur noch von zugelassenen Vertragsärzten, von Kliniken und von gemeinnützigen Trägern gegründet werden. „Die Gründung ist nur in der Rechtsform einer Personengesellschaft oder einer Gesellschaft mit beschränkter Haftung möglich," so der neu eingefügte Satz in $\mathrm{Pa}$ - ragraf 95. Für bestehende Einrichtungen soll aber Bestandsschutz gelten. Leutloff sieht in der Beschränkung der MVZ-Gründer allerdings eher Kosmetik: „Auch Kliniken dürfen MVZ gründen, und gerade Privatkliniken gehören häufig zu Aktiengesellschaften." Bei der ärztlichen Leitung müssen sich allerdings nach den derzeitigen Plänen alle MVZ den neuen Regeln beugen, auch MVZ in Klinikhand.

Eine wichtige Neuerung ist ein Vorkaufsrecht für niedergelassene Ärzte: Wenn sich ein MVZ und ein Vertragsarzt um einen freien Vertragsarztsitz bewerben, hat der Vertragsarzt das Vorkaufsrecht - auch wenn der Sitz in eine Gemeinschaftspraxis eingebracht wird. „Insgesamt wird mit den neuen Regeln zu MVZ die Freiberuflichkeit gestärkt", so die abschließende Analyse Leutloffs. Das kommt den Bestrebungen der Standesbank entgegen, in Kooperationen oder ärztlichen Zentren Strukturen zu fördern, in denen die Ärzte als Freiberufler das Sagen haben.

Antonia von Alten und Hauke Gerlof, Neu-Isenburg

Haben Sie Fragen?

Wenn Sie erfahren wollen, was die Neuerungen im GKV-Versorgungsstrukturgesetz für Ihre persönliche Situation bedeuten könnten, leiten wir Ihre Fragen gerne an die Experten der apoBank weiter. Schreiben Sie uns unter: wi@springer.com (Stichwort: GKV-VStG)

Ein Service der Deutschen Apotheker- und Ärztebank

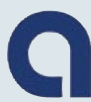

